OPEN ACCESS

Edited by:

Christoph Schaniel,

Icahn School of Medicine at Mount

Sinai, United States

Reviewed by:

Sulev Kõks,

University of Tartu, Estonia

Fanxiao Liu,

Shandong Provincial Hospital, China

*Correspondence:

Hui L

lihuix@csu.edu.cn

Specialty section:

This article was submitted to

Stem Cell Research,

a section of the journal

Frontiers in Cell and Developmental

Biology

Received: 04 August 2021 Accepted: 22 September 2021

Published: 18 October 2021

Citation

Xiao B, Liu L, Chen Z, Li A, Xia Y, Wang $P$, Xiang $C$, Zeng $Y$ and $\mathrm{Li} H$

(2021) A Novel Overall Survival

Prediction Signature Based on

Cancer Stem Cell-Related Genes

in Osteosarcoma.

Front. Cell Dev. Biol. 9:753414. doi: 10.3389/fcell.2021.753414

\section{A Novel Overall Survival Prediction Signature Based on Cancer Stem Cell-Related Genes in Osteosarcoma}

\author{
Bo Xiao ${ }^{1,2}$, Liyan Liu'1,2, Zhuoyuan Chen ${ }^{1,2}$, Aoyu Li, ${ }^{1,2}$, Yu Xia ${ }^{1,2}$, Pingxiao Wang ${ }^{1,2}$, \\ Cheng Xiang ${ }^{1,2}$, Yi Zeng ${ }^{1,2}$ and Hui Li, ${ }^{1,2 *}$ \\ ${ }^{1}$ Department of Orthopedics, The Second Xiangya Hospital, Central South University, Changsha, China, ${ }^{2}$ Orthopedic \\ Biomedical Materials Engineering Laboratory of Hunan Province, Changsha, China
}

Background: Osteosarcoma is the most general bone malignancy that mostly affects children and adolescents. Numerous stem cell-related genes have been founded in distinct forms of cancer. This study aimed at identifying a stem cell-related gene model for the expected assessment of the prognosis of osteosarcoma patients.

Methods: We obtained the genes expression data and relevant clinical materials from Therapeutically Applicable Research to Generate Effective Treatments (TARGET) and Gene Expression Omnibus (GEO) databases. We identified differentially expressed genes (DEGs) from the GEO dataset, whereas prognostic stem cell-related genes were obtained from the TARGET database. Subsequently, univariate, LASSO and multivariate Cox regression analyses were applied to establish the stem cell-related signature. Finally, the prognostic value of the signature was validated in the GEO dataset.

Results: Twenty-five genes were prognostic ferroptosis-related DEGs. Consequently, we identified eight stem cell-related genes as a signature of prognosis of osteosarcoma patients. Then, the Kaplan-Meier (K-M) curve, the AUC value of ROC, and Cox regression analysis verified that the eight stem cell-related gene model were a new and substantial prognostic marker independent of other clinical traits. Moreover, the nomogram on the foundation of risk score and other clinical traits was established for predicting the survival rate of osteosarcoma patients. Biological function analyses displayed that tumor related pathways were affluent.

Conclusion: The expression level of stem cell-related genes offers novel prognostic markers as well as underlying therapeutic targets for the therapy and prevention of osteosarcoma.

Keywords: osteosarcoma, stem cells, gene, overall survival, prognosis, signature

\section{BACKGROUND}

Osteosarcoma (OS) is the most widespread main bone malignancy in children and young adults (Rothzerg et al., 2020) as well as makes up for nearly two-thirds of the primary bone malignancies diagnosed in the first 20 years of one's life (Yan et al., 2016; Chen et al., 2019). The combination of surgical resection skills and multiagent chemotherapy has extremely enhanced the prognosis of 
osteosarcoma patients (Bielack et al., 2002). Other studies have shown that over the last 30 years, neoadjuvant chemotherapy and surgical techniques have developed, and the survival rate of 5 years in these patients has increased to $\sim 70 \%$ (Bielack et al., 2002; Niu et al., 2019; Wakamatsu et al., 2019). On the other hand, the overall survival rate of 5 years in cases diagnosed with early pulmonary metastasis is less than $20 \%$. Besides, another study shows that $20 \%$ of patients without early pulmonary metastasis have local recurrence or will eventually develop pulmonary metastasis (Bishop et al., 2016). However, the fact that these patients develop resistance to chemotherapy is a major challenge. Therefore, it is imperative to explore novel prognostic models to further improve the survival of osteosarcoma patients.

Cancer stem cells (CSCs), a small, special class of cells inside tumors, owns the capability of self-renewal and differentiation of different cell types that assemble the whole tumor (Reya et al., 2001). CSCs are tumor-derived cells that mutate from stem cells of adults and produce distinct subtypes of tumor cells via self-renewal and differentiation (van der Heijden and Vermeulen, 2019). CSCs were associated with the development, recurrence, metastasis, and resistance of cancers and facilitate tumor progression (Dawood et al., 2014; Nassar and Blanpain, 2016). The capability of CSCs to originate and maintain the proliferation of cancer cells is an essential factor for metastasis. CSCs are heterogeneous and own subgroups with metastatic ability (Nandy and Lakshmanaswamy, 2017; Aydemir Coban and Sahin, 2018). Besides, CSCs can stay in prolonged dormancy and have varieties of molecular mechanisms of chemotherapy resistance (Lytle et al., 2018). As CSCs act as crucial roles in tumor progression, CSC biomarkers and related pathways may become the prognostic biomarkers and underlying targets for treatment of osteosarcoma patients.

Herein, we mined the expression data of genes and the relevant clinical information of osteosarcoma samples, and CSC-related genes from the opening databases. Next, the prognostic CSC-related gene signature was built in the Therapeutically Applicable Research to Generate Effective Treatments (TARGET) cohort and then evaluated it in the Gene Expression Omnibus (GEO) dataset. Last, we explored the potential mechanisms underlying CSC-related genes using a functional enrichment analysis.

\section{MATERIALS AND METHODS}

\section{Data Collection}

The TARGET database was applied to acquire the mRNA expression data and corresponding clinical features of osteosarcoma samples. The information of clinical follow-up in this cohort is shown in Table $\mathbf{1 .}$

The mRNA expression of GSE33383 and GSE21257 were mined from the GEO database; the package of "limma" was applied for quality control and normalization. The platform of GSE33383 and GSE21257 is GPL10295. The expression value for genes with numerous probes was computed as the average of the probes. The dataset of GSE33383 contains 15 mesenchymal stem cells/osteoblasts and 84 samples of osteosarcoma tissues, while the dataset of GSE21257 contains 53 patients of osteosarcoma, serving as a validating set. The clinical features of GSE21257 are shown in Table 1.

The CSC-related pathways were mined from the Molecular Signature Database v 7.4 (MSigDB). Altogether, 413 CSC-related genes were classified from the 31 pathways in the Gene Ontology (GO) and reactome databases (Table 2).

\section{Establishment of the Cancer Stem Cell-Related Gene Model in the Therapeutically Applicable Research to Generate Effective Treatments Database}

To explore the differentially expressed genes (DEGs) among normal and osteosarcoma samples with the FDR value $<0.05$, we employed the "limma" R package present in the GSE33383 cohort. Next, we employed univariate Cox analysis to compute the prognostic values of CSC-related genes $(p<0.05)$ for the DEGs in the TARGET database (Rothzerg et al., 2021). Then, the LASSO regression analysis was applied for screening the survival-related genes for the outcomes of univariate Cox regression analysis utilizing the "glmnet" package. Finally, to optimize the model, we applied the multivariate regression analysis, which constructs the prognostic signature on the foundation of the expression and corresponding coefficients of CSC-related genes. The formula of risk score was as follows: risk score $=\Sigma i$ coefficient $($ gene $i) \times$ expression $($ gene $i$ ) Consequently, the median value of risk score was obtained employing the "Survminer" package. Here, the patients of osteosarcoma were classified into two risk groups (low and high). Moreover, the Kaplan-Meier (K-M) curve and timedependent receiver-operating characteristic (ROC) curve were applied to assess the clinical prognostic risk score capacity. Furthermore, the curves of survival score and survival status as well as heatmap were employed to illustrate the distribution of osteosarcoma patients in two groups (high risk and low risk). Last, we used different variables such as gender, age, tumor site, risk score, and metastasis to evaluate whether the risk score is an independent prognostic biomarker for patients of osteosarcoma, using uni- and multivariate Cox regression analysis.

\section{Establishment and Calibration of Nomogram}

A nomogram was employed for predicting the survival of osteosarcoma patients. Here, different variables such as age, gender, risk score, race, tumor site, and metastasis were employed to construct the nomogram utilizing the R packages "rms" and "survival." Finally, the resulting calibration curve was applied to assess the accurateness of the nomogram between different groups of patients.

\section{Functional Enrichment Analysis}

The GO that comprises biological processes (BP), cellular components (CC), and molecular functions (MF), as well as KEGG analyses were achieved in two risk groups (low and high) 
TABLE 1 | Clinical features of the osteosarcoma samples in the Therapeutically Applicable Research to Generate Effective Treatments (TARGET) and Gene Expression Omnibus (GEO) databases.

\begin{tabular}{|c|c|c|c|}
\hline Variables & TARGET $(n)$ & GEO $(n)$ & \\
\hline \multicolumn{4}{|l|}{ Age (year) } \\
\hline$\leq 16$ & 56 & 25 & \\
\hline$>16$ & 39 & 28 & \\
\hline \multicolumn{4}{|l|}{ Gender } \\
\hline Female & 40 & 19 & \\
\hline Male & 55 & 34 & \\
\hline Race & & Huvos grade & \\
\hline White & 55 & 1 & 13 \\
\hline Asian & 6 & 2 & 16 \\
\hline Black & 9 & 3 & 13 \\
\hline Other & 1 & 4 & 5 \\
\hline Unknown & 24 & Unknown & 6 \\
\hline \multicolumn{4}{|l|}{ Metastasis } \\
\hline Yes & 23 & 34 & \\
\hline No & 72 & 19 & \\
\hline \multicolumn{4}{|l|}{ Survival status } \\
\hline Alive & 57 & 30 & \\
\hline Death & 38 & 23 & \\
\hline \multicolumn{4}{|c|}{ Primary tumor sites } \\
\hline Leg/foot & 83 & 44 & \\
\hline Arm/hand & 7 & 8 & \\
\hline Pelvis & 4 & / & \\
\hline Other/unknown & 1 & 1 & \\
\hline
\end{tabular}

utilizing the "clusterProfiler" R package with $|\log 2 \mathrm{FC}|>1$ and FDR value $<0.05$.

\section{Statistical Analysis}

Here, the DEGs among normal and osteosarcoma cohorts were calculated using the Wilcox test. The K-M curve was employed to compare the overall survival between the two risk groups using the Chi-square test. Subsequently, Cox regression analyses were carried out to study independent prognostic variables. The $\mathrm{R}$ statistical software (Version 4.0.1) was employed to execute all statistical analyses.

\section{RESULTS}

Figure 1 demonstrates a flow chart that explains the study. In this study, we mined data for a total of 95 patients with osteosarcoma and who had complete clinical information from the TARGET database, 15 normal samples, and 84 osteosarcoma samples in GSE33383 from the GEO database. Besides, the GSE21257 served as the validating cohort.

\section{Distinction of Prognostic Cancer Stem Cell-Related Differentially Expressed Genes}

Here, we explored the mRNA expression of 413 CSC-related genes in the GSE33383 dataset using the "limma" package in
TABLE 2 | Pathways associated with cancer stem cells in the Gene Ontology (GO) and reactome databases.

\begin{tabular}{|c|c|c|}
\hline Stem cell function related pathways & ID & Gene number \\
\hline GO: Stem cell division & GO:0017145 & 25 \\
\hline $\begin{array}{l}\text { GO: Somatic stem cell population } \\
\text { maintenance }\end{array}$ & GO:0035019 & 71 \\
\hline GO: Hematopoietic stem cell migration & GO:0035701 & 8 \\
\hline GO: Somatic stem cell division & GO:0048103 & 9 \\
\hline GO: Stem cell differentiation & GO:0048863 & 251 \\
\hline GO: Stem cell fate commitment & GO:0048865 & 6 \\
\hline GO: Hematopoietic stem cell differentiation & GO:0060218 & 88 \\
\hline GO: Hematopoietic stem cell homeostasis & GO:0061484 & 15 \\
\hline GO: Hematopoietic stem cell proliferation & GO:0071425 & 25 \\
\hline $\begin{array}{l}\text { GO: Mesenchymal stem cell maintenance } \\
\text { involved in nephron morphogenesis }\end{array}$ & GO:0072038 & 5 \\
\hline GO: Stem cell proliferation & GO:0072089 & 64 \\
\hline GO: Regulation of stem cell proliferation & GO:0072091 & 41 \\
\hline GO: Mesenchymal stem cell differentiation & GO:0072497 & 9 \\
\hline $\begin{array}{l}\text { GO: Neuronal stem cell population } \\
\text { maintenance }\end{array}$ & GO:0097150 & 19 \\
\hline GO: Mesenchymal stem cell proliferation & GO:0097168 & 8 \\
\hline $\begin{array}{l}\text { GO: Regulation of hematopoietic stem cell } \\
\text { proliferation }\end{array}$ & GO:1902033 & 11 \\
\hline $\begin{array}{l}\text { GO: Positive regulation of hematopoietic } \\
\text { stem cell proliferation }\end{array}$ & GO:1902035 & 6 \\
\hline $\begin{array}{l}\text { GO: Regulation of hematopoietic stem cell } \\
\text { differentiation }\end{array}$ & GO:1902036 & 74 \\
\hline $\begin{array}{l}\text { GO: Negative regulation of hematopoietic } \\
\text { stem cell differentiation }\end{array}$ & GO:1902037 & 5 \\
\hline $\begin{array}{l}\text { GO: Negative regulation of stem cell } \\
\text { population maintenance }\end{array}$ & GO:1902455 & 7 \\
\hline $\begin{array}{l}\text { GO: Positive regulation of stem cell } \\
\text { population maintenance }\end{array}$ & GO:1902459 & 7 \\
\hline $\begin{array}{l}\text { GO: Positive regulation of mesenchymal } \\
\text { stem cell proliferation }\end{array}$ & GO:1902462 & 5 \\
\hline $\begin{array}{l}\text { GO: Regulation of somatic stem cell } \\
\text { population maintenance }\end{array}$ & GO:1904672 & 8 \\
\hline GO: Regulation of stem cell division & GO:2000035 & 10 \\
\hline $\begin{array}{l}\text { GO: Regulation of stem cell population } \\
\text { maintenance }\end{array}$ & GO:2000036 & 31 \\
\hline $\begin{array}{l}\text { GO: Negative regulation of stem cell } \\
\text { proliferation }\end{array}$ & GO:2000647 & 9 \\
\hline $\begin{array}{l}\text { GO: Positive regulation of stem cell } \\
\text { proliferation }\end{array}$ & GO:2000648 & 22 \\
\hline GO: Regulation of stem cell differentiation & GO:2000736 & 112 \\
\hline $\begin{array}{l}\text { GO: Negative regulation of stem cell } \\
\text { differentiation }\end{array}$ & GO:2000737 & 21 \\
\hline $\begin{array}{l}\text { GO: Positive regulation of stem cell } \\
\text { differentiation }\end{array}$ & GO:2000738 & 18 \\
\hline $\begin{array}{l}\text { GO: Regulation of mesenchymal stem cell } \\
\text { differentiation }\end{array}$ & GO:2000739 & 6 \\
\hline $\begin{array}{l}\text { Reactome transcriptional Regulation of } \\
\text { Pluripotent Stem Cells }\end{array}$ & R-HSA-452723 & 31 \\
\hline
\end{tabular}

$\mathrm{R}$ statistical software. Among the normal and osteosarcoma samples, we used a threshold of FDR value $<0.05$ and identified 210 differentially expressed CSC-related genes (Figures 2A,B). Next, we computed the prognostic values of CSC-related DEGs in the TARGET database utilizing the univariate Cox regression 


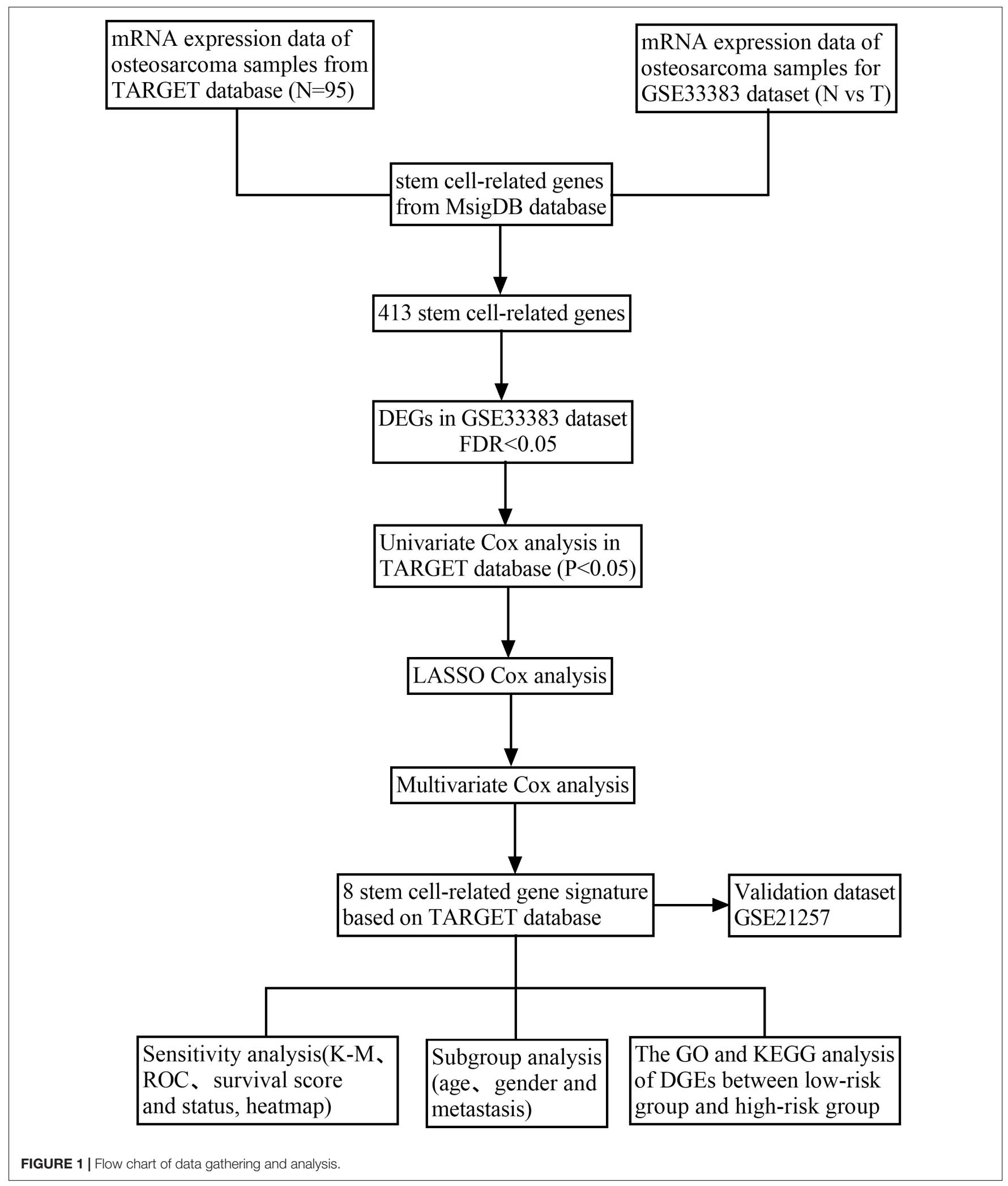

analysis. As illustrated in Figure 2C, we observed that altogether, 25 CSC-related genes correlated with the overall survival of osteosarcoma $(p<0.05)$. Hence, the 25 genes were prognostic
CSC-related DEGs. Besides, the protein-protein interaction (PPI) network of the 25 prognostic CSC-related DEGs are shown in Figure 2D. 

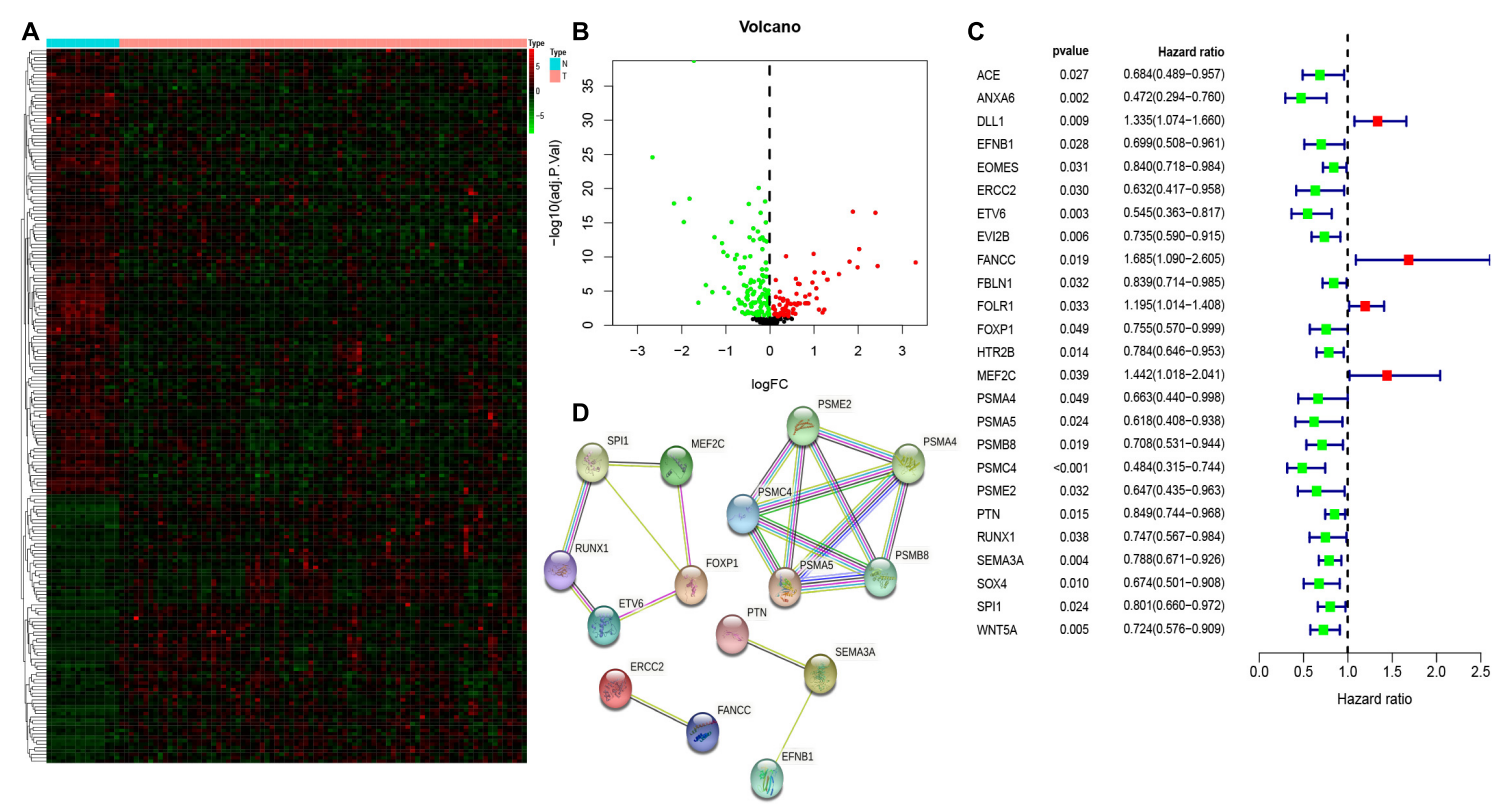

FIGURE 2 | The heatmap (A) and volcano (B) of differentially expressed genes (DEGs) between osteosarcoma and normal tissues/cells. The univariate Cox regression analysis of 25 prognostic stem cell-related DEGs (C). The protein-protein interaction (PPI) network showed the interactions of 25 prognostic stem cell-related DEGs (D).
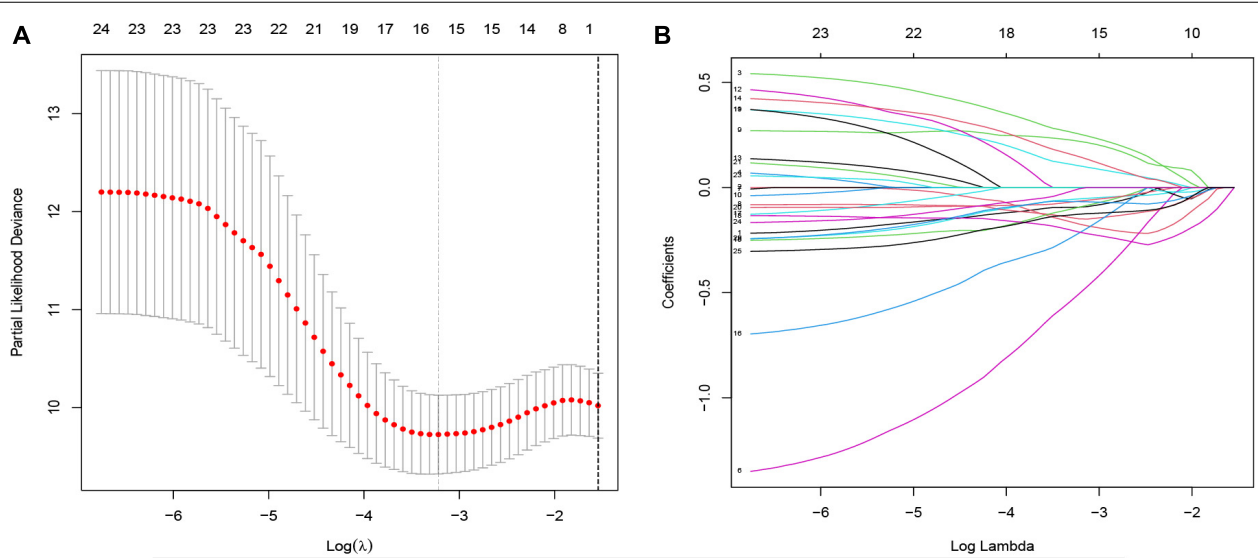

C Hazard ratio

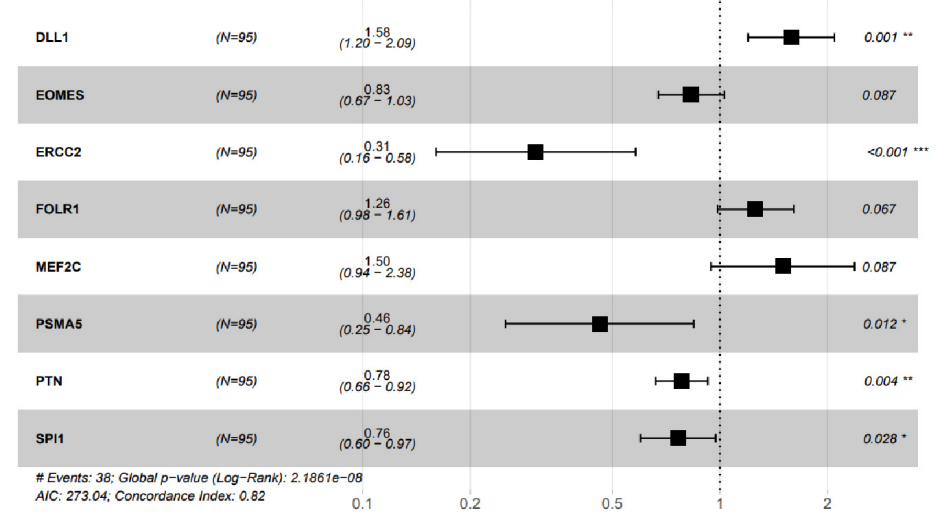

FIGURE 3 | Establishment of stem cell-related gene prognostic signature. (A,B) The LASSO Cox analysis determined 15 prognostic genes. (C) The forest map of multivariate Cox analysis. ${ }^{\star} P<0.05$; ${ }^{\star \star} P<0.01$; ${ }^{\star \star \star} P<0.001$. 

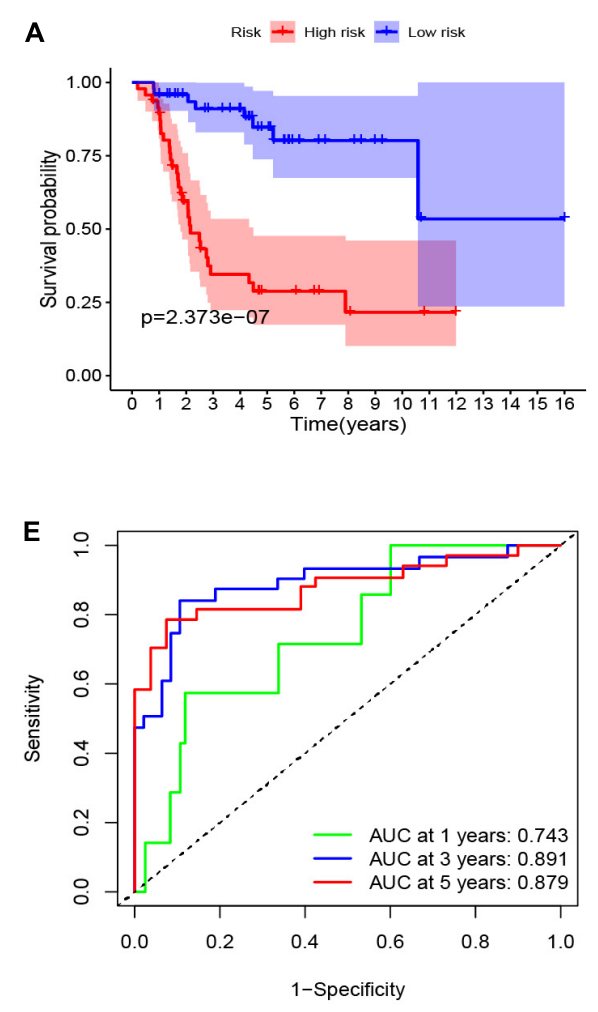

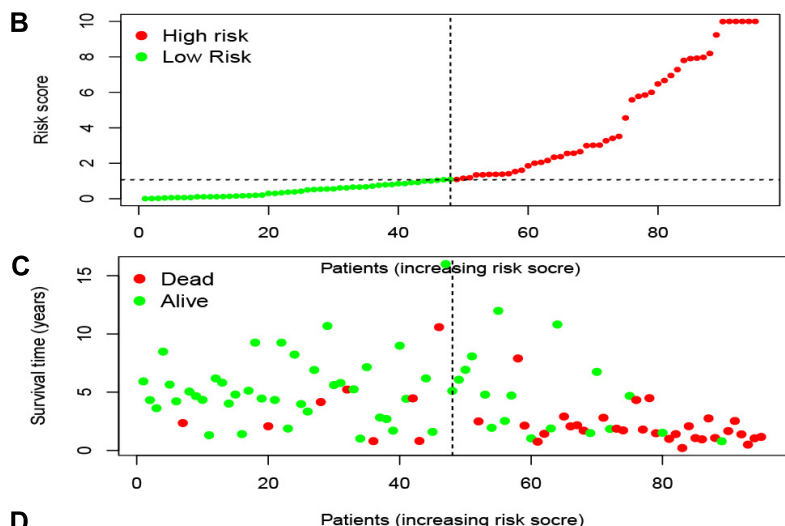

D

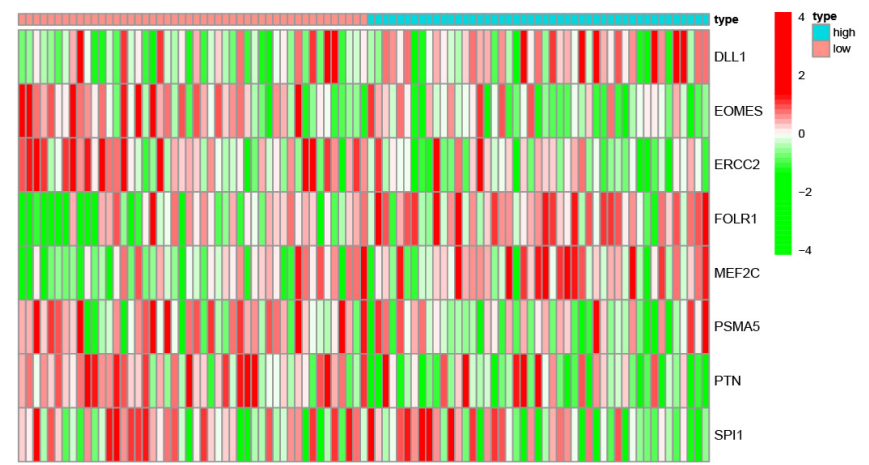

FIGURE 4 | (A) The Kaplan-Meier (K-M) curve of the eight-gene signature in the TARGET cohort. The risk score (B), survival time and status (C), as well as the expression of the eight genes (D) in the TARGET set. (E) The receiver-operating characteristic (ROC) curve and AUC value of the eight-gene signature in the TARGET set.

\section{Establishment and Evaluation of the Prognostic Cancer Stem Cell-Related Gene Signature in the Therapeutically Applicable Research to Generate Effective Treatments}

In this study, we then applied LASSO regression analysis on these 25 CSC-related genes, and 16 genes were selected as candidate genes via relative regression coefficient (Figures $3 \mathbf{A}, \mathbf{B}$ ). Finally, the multivariate Cox analysis was applied, and eight genes [delta-like ligand 1 (DLL1), EOMES, ERCC2, folate receptor 1 (FOLR1), myocyte enhancer factor 2C (MEF2C), PSMA5, PTN, and SPI1] with a minimum Akaike's information criterion (AIC) value of 273.04 were distinguished to establish the risk signature (Figure 3C). Subsequently, the risk scores were computed on the foundation of the expression level of eight CSC-related prognostic genes, and the corresponding value of coefficient was derived from the multivariate Cox model. Risk score $=(0.45792456 \times$ DLL1 expression $)+$ $(-0.1851977 \times$ EOMES expression $)+(-1.1867301 \times$ ERCC2 expression $)+(0.22938571 \times$ FOLR1 expression $)+$ $(0.40383058 \times$ MEF2C expression $)+(-0.775786 \times$ PSMA5 expression $)+(-0.246729 \times$ PTN expression $)+(-0.2701665 \times$ SPI1 expression).

Consequently, the patients were separated into two risk groups (low and high) on the foundation of the median risk score. As demonstrated in Figure 4A, the K-M curves revealed that the high-risk group had an extremely poorer survival rate than the patients in the low-risk group ( $p=2.373 \mathrm{e}-07)$, showing that the risk score has a valid prognosis value. The risk score and survival status of all osteosarcoma samples were presented in the form of risk plot and scatter, respectively. Moreover, as shown in Figures 4B,C, the mortality and risk coefficient of low-risk patients were lower compared with that of high-risk patients. The assignation of these eight gene expressions in the subgroups of the risk score are shown in Figure 4D. Besides, we used ROC curves to appraise the precision of these eight CSC-related gene signature in forecasting the overall survival of osteosarcoma patients. The AUC values of 1,3 , and 5 years were $0.743,0.891$, and 0.879 , respectively (Figure $4 \mathrm{E}$ ). This shows an outstanding potentiality of our eight CSC-related gene model in predicting.

\section{Assessment of Eight Cancer Stem Cell-Related Gene Model as Independent Prognostic Factor}

Here, we employed Cox regression analysis tests to examine whether the eight CSC-related genes signature was an independent prognostic variable for other clinical traits, like age, gender, race, tumor site, and metastasis. The univariate Cox regression analysis illustrated that the risk score was an independent variable for predicting the prognosis of 

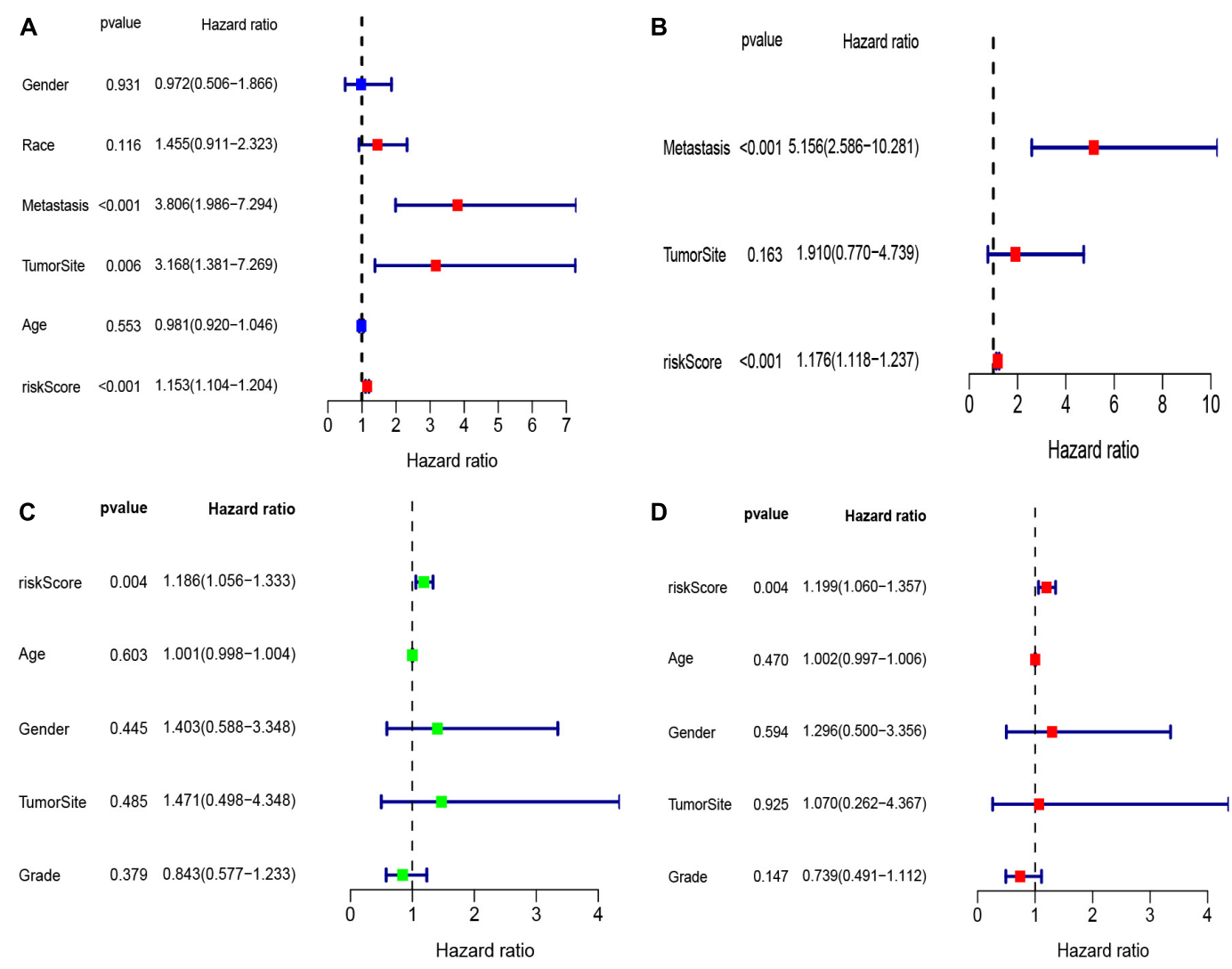

FIGURE 5 | The forest maps of univariate and multivariate Cox regression analysis in the TARGET (A,B) and GEO (C,D) datasets.

A Patients with age $<=16$

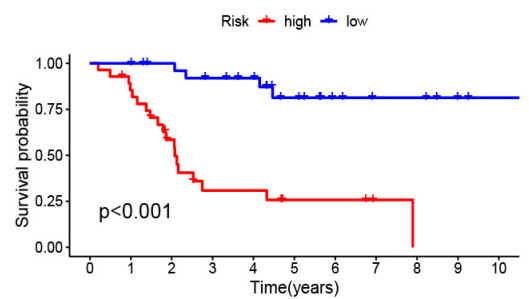

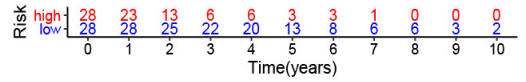

B Patients with age $>16$

Risk + high - low

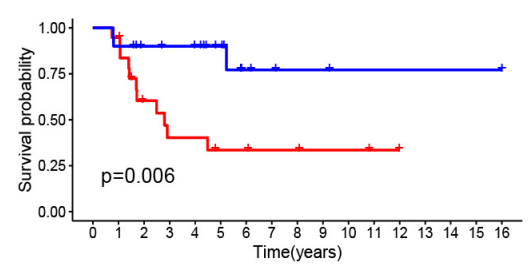

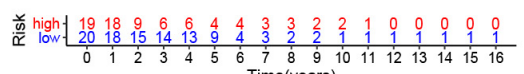

C Patients with Female

Risk - high + low

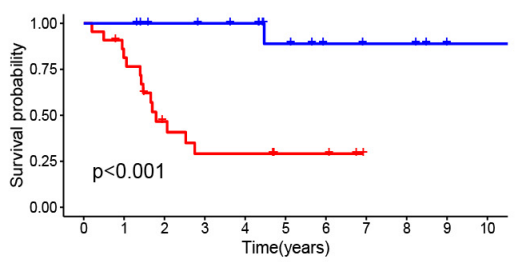

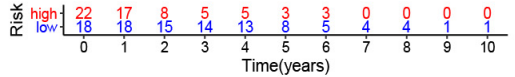

D Patients with Male

Risk + high - low

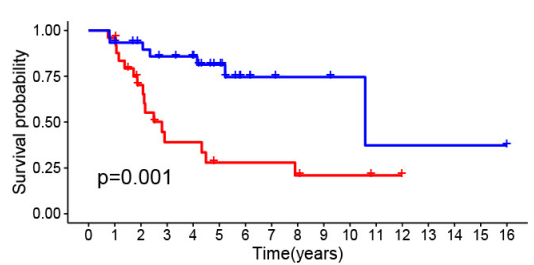

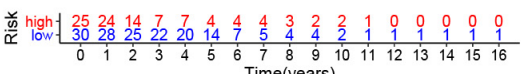

E Patients with No

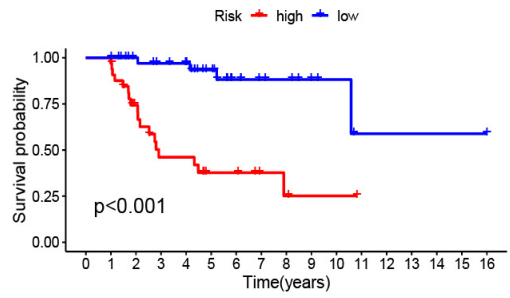

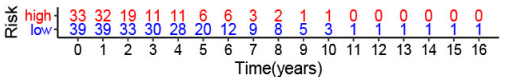

F Patients with Yes

Risk + high — low

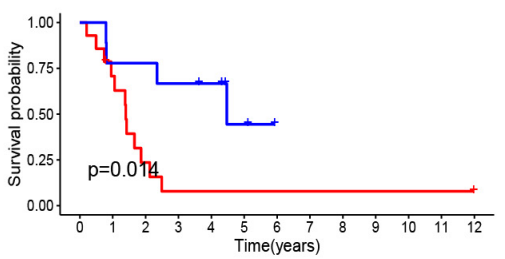

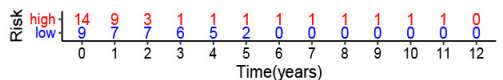

FIGURE 6 | The K-M curves for the two groups (high risk vs. low risk) assigned by clinical factors, comprising age (A,B), gender (C,D), and metastasis (E,F). 

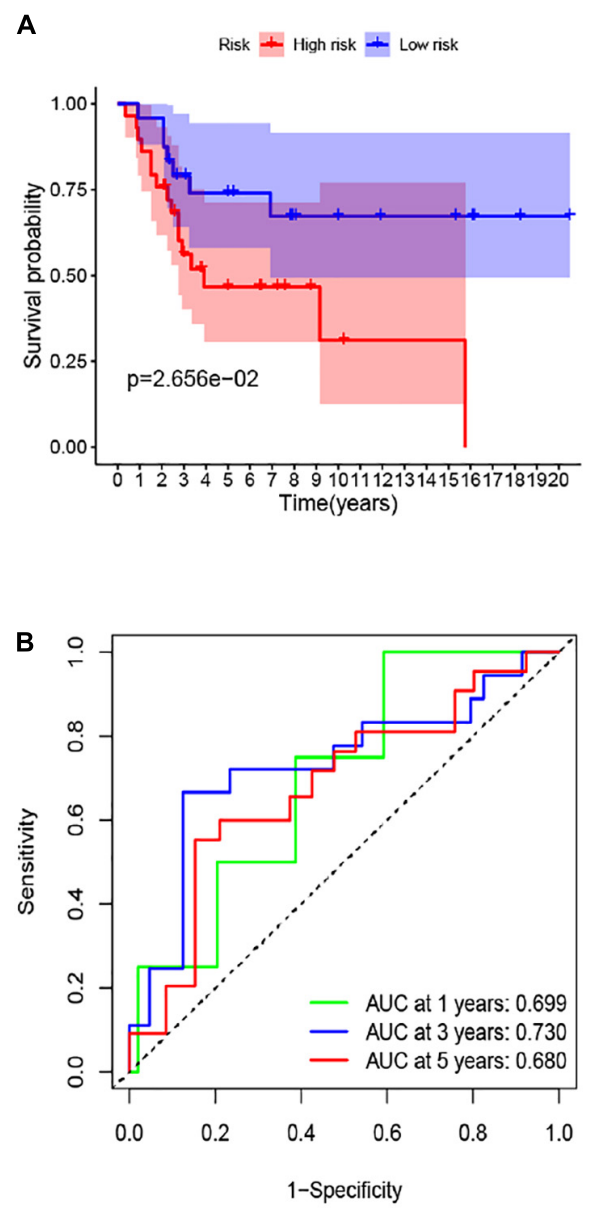
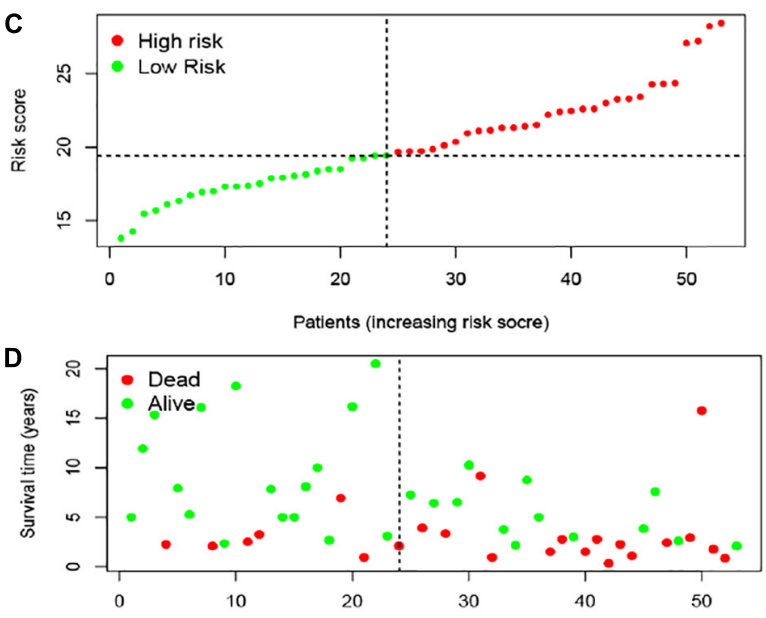

$\mathbf{E}$

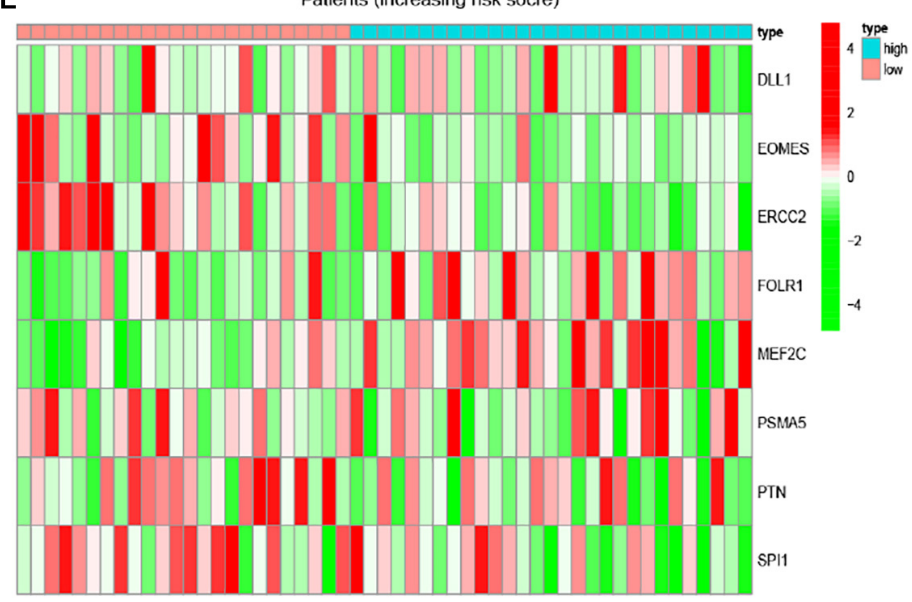

FIGURE 7 | (A) The K-M curve of the eight-gene signature in the GEO test cohort. (B) The ROC curve and AUC value of the eight-gene signature in the GEO set. (C-E) The risk score, survival time, and status as well as the expression of the eight genes in the GEO set.

osteosarcoma patients $(\mathrm{HR}=1.157,95 \% \mathrm{CI}: 1.106-1.211$, and $p<0.001$, Figure 5A). The multivariate Cox regression analysis also showed that the risk score was an independent prognostic factor $(p<0.001, \mathrm{HR}=1.191,95 \% \mathrm{CI}: 1.125-1.260$, Figure 5B). Besides, to explore the prognostic value of the signature in osteosarcoma patients sorted by clinical variables, we divided the samples into distinct subgroups according to age, gender, and metastasis. As shown in Figure 6, the overall survival of osteosarcoma patients in the low-risk group was remarkably better than that of the high-risk group $(p<0.05)$.

\section{Verification of the Risk Signature in the Gene Expression Omnibus Database}

To validate the robustness of these eight CSC-related gene signature, we employed another independent cohort GSE21257. Based on the formula of the risk score above, the patients of the GSE21257 cohort were assigned into two risk groups, with 29 samples in the high-risk group and 24 samples in the low-risk group. Similar to previous results, the result of the $\mathrm{K}-\mathrm{M}$ curve also verified that patients in the low-risk group had seriously longer overall survival than those in the high-risk group (Figure 7A). The AUC values of the CSC-related gene signature for predicting the 1-, 3-, and 5-year survival were 0.699, 0.730, and 0.680 , respectively (Figure $7 \mathbf{B}$ ). The heatmap illustrated the gene expression levels of signature in the two risk groups of the validation set. The risk score and survival status of osteosarcoma samples in GSE21257 were also displayed in the form of a risk plot and scatter, respectively (Figures 7C-E). Besides, the univariate (Figure 5C) and multivariate (Figure 5D) Cox analysis explored that the risk score was also an independent prognostic biomarker for patients of osteosarcoma in the GSE21257. All of the results confirmed that the eight CSC-related gene signature was valid and robust.

\section{Establishment of Nomogram}

To forecast the 1-, 3-, and 5-year overall survival of each patient, we performed a nomogram on the foundation of the TARGET dataset. As shown in Figure 8A, the expression signature for risk score, age, gender, race, tumor site, and metastasis were employed as variables. Notably, as demonstrated 
A

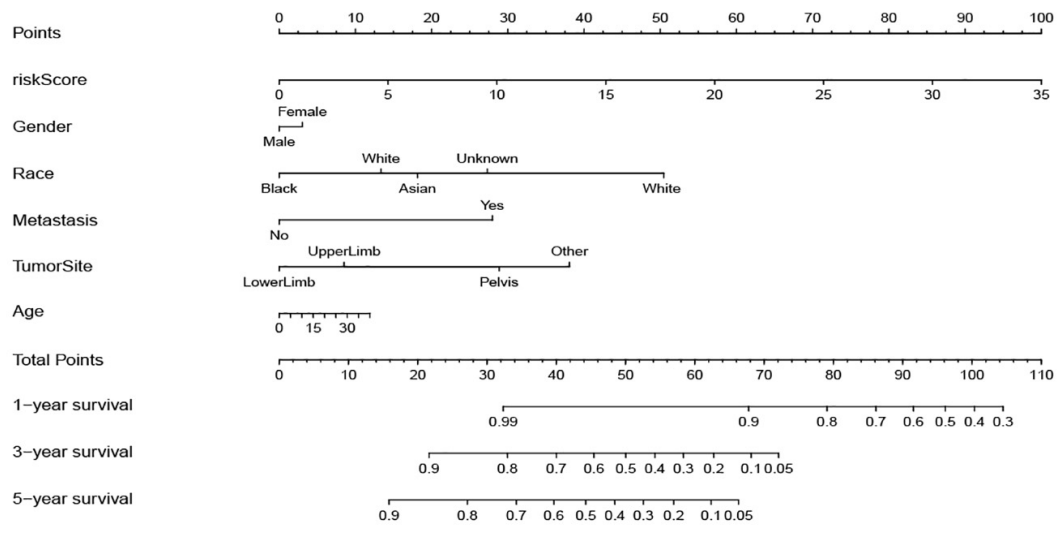

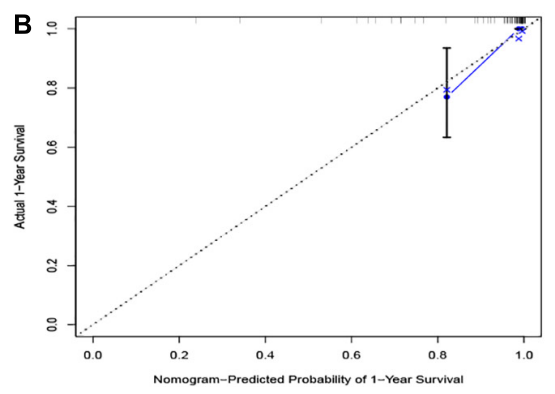
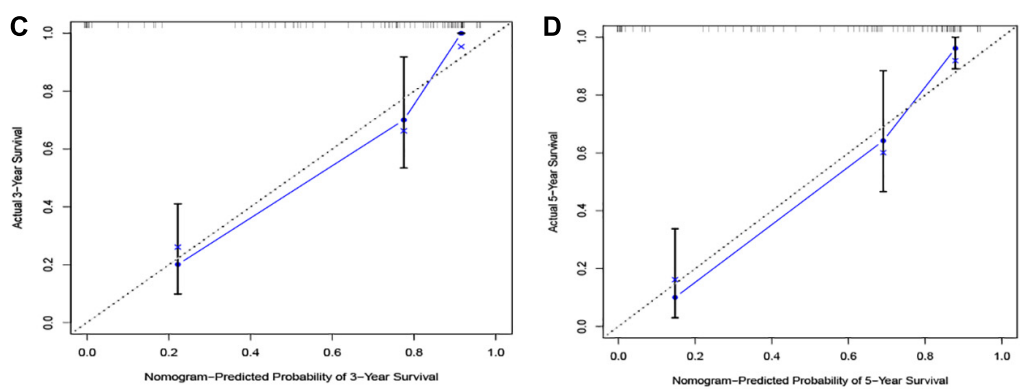

FIGURE 8 | (A) The nomogram based on the TARGET database. The calibration curves were applied to predict the 1- (B), 3- (C), and 5-year (D) survival in the TARGET cohort.

in Figures 8B-D, the calibration curve of the 1-, 3-, and 5-year overall survival were obtained, and they compared well with the ideal model.

\section{Risk Score and Potential Biological Functions}

To explore the underlying biological functions between the two risk groups, we identified the DEGs among the two groups. The top 10 GO terms and KEGG of 1,386 DEGs are shown (Figure 9). The most enriched GO terms were CC such as focal adhesion, cell-substrate junction, endosome membrane, collagen-containing extracellular matrix, cell leading edge, and so on; MF, for example, cell adhesion molecule binding, nucleoside binding, ribonucleoside binding, GTP binding, and so on; and BP such as regulation of cell morphogenesis, autophagy, regulation of Wnt signaling pathway, ephrin receptor signaling pathway, and so on (Figure 9A). The KEGG pathway of 1,386 DEGs were mainly related to the Ras signaling pathway, MAPK signaling pathway, chemokine signaling pathway, Rap1 signaling pathway, apoptosis, and so on (Figure 9B).

\section{DISCUSSION}

Osteosarcoma is a common malignant tumor of the bone with an elevated tendency of invasion and metastasis (Chen et al., 2021). Although there are multiple advances in comprehensive therapies for osteosarcoma, the prognosis of osteosarcoma patients is still poor. Thus, exploring the valid prognostic biomarkers and potential therapeutic targets is very critical for improving the prognosis of osteosarcoma. CSCs are a set of heterogeneous cells that own distinct differentiation abilities. Very little subpopulations of cancer cell have properties of stem cell in tumor tissues, but the potential of differentiation and proliferation as well as the abilities of self-renewal are the derived factors for tumorigenesis, development, invasion, recurrence, metastasis, and resistance. In this study, we explored the expression profile of 413 CSC-related genes between normal and osteosarcoma cohorts and obtained 210 genes that were differentially expressed among normal and osteosarcoma tissues in the GEO dataset. Then, we employed the univariate Cox regression analysis to explore the correlation between 210 differentially expressed CSC-related genes and the prognosis of osteosarcoma patients. Finally, an eight CSC-related gene signature was constructed by LASSO and multivariate Cox analysis. The curves of K-M and ROC confirmed that the predictive performance of the signature is stable and consistent with the TARGET internal and GEO external validation sets, and it was remarkably associated with the clinicopathological features and matrix score. What is more, the Cox analyses indicated that the eight-gene signature had an independent predictive capability for prognosis in two distinct cohorts. All of the results showed that the eight-gene signature had a significant potential in clinical application. 


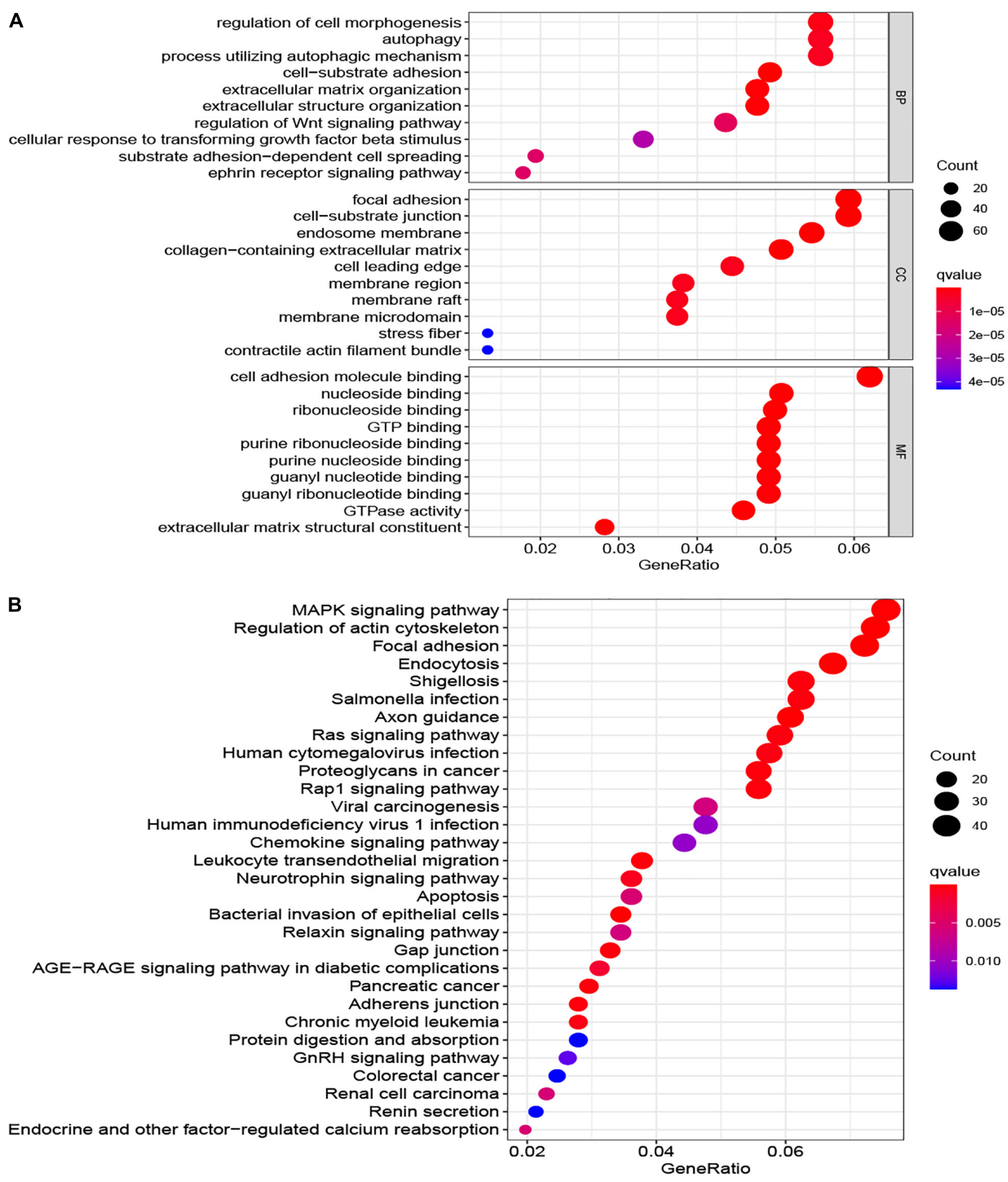

FIGURE 9 | The most significant outcomes of GO (A) and KEGG (B) analysis of DEGs between high- and low-risk group.

In the eight-gene signature identified and validated on the foundation of CSC-related genes, DLL1, FOLR1, and MEF2C were risk variables, while EOMES, ERCC2, PSMA5, PTN, and SPI1 were protective factors. Delta-like ligand 1 was highly expressed in metastatic osteosarcoma cells compared with osteoblasts and non-metastatic osteosarcoma cells (Hughes, 2009). Besides, Pu et al. (2017) found that the expression of DLL1 is negatively associated with multichemoresistance of osteosarcoma. MiR-34a-5p enhances multichemoresistance of osteosarcoma by repressing DLL1. Folate receptor 1 is overexpressed in varieties of solid tumors (Necela et al., 2015; Huang et al., 2018; Kim et al., 2018) and is closely associated with the prognoses of multiple malignant tumors, such as triplenegative breast cancer (Ginter et al., 2017), ovarian cancer (Kobel et al., 2014), and non-small-cell lung cancer (Iwakiri et al., 2008). Myocyte enhancer factor $2 \mathrm{C}$ was overexpressed in hepatocellular carcinoma. MEF2C-mediated VEGF-induced vasculogenic mimicry, angiogenesis, and invasion, as well as inhibition of $\beta$-catenin-induced tumor growth (Bai et al., 2015). Besides, MEF2C was upregulated in gefitinib-resistant cancer tissues and associated with gefitinib resistance in hepatic cancer cells (Zhang et al., 2018). He et al. (2021) found that EOMES was down expressed in tissue samples and related to the advanced stage and poor prognosis of hepatocellular carcinoma. Moreover, EOMES 
is associated with the disease-free survival time in gastric cancer (Cui et al., 2020) and also associated with better overall survival in triple-negative breast cancer (Wang et al., 2018). The gene polymorphisms of ERCC2 and ERCC1 were related to chemotherapy sensitivity and overall survival in osteosarcoma patients (Cao et al., 2015). PSMA5 is dysregulated in prostate cancer and associated with bortezomib resistance as well as disease-free survival (Fu et al., 2019). PTN is highly expressed in osteosarcoma, and by target PTN, miR-627-3p inhibits proliferation and migration of osteosarcoma cells (He et al., 2019). LncRNA OIP5-AS1 is related to doxorubicin resistance of osteosarcoma via mediating miR-137-3p/PTN axis (Sun et al., 2020). Besides, the whole transcriptome RNA-seq also proved that PTN is differentially expressed in osteosarcoma (Reimann et al., 2014). Bioinformatics analysis showed that SPI1 was a prognosis-related gene in esophageal squamous cell carcinoma (Ho et al., 2017; Zhang et al., 2020). Most of these genes were related to prognosis in the other cancers, but whether these genes relate to the prognosis of osteosarcoma by altering the molecular mechanism of stem cells still is unclear because only a few correlational researches about these genes have been described.

Next, we assessed the association between risk score, gender, age, and metastasis in osteosarcoma patients through Cox regression analysis. All of the outcomes demonstrated that the risk score could be the independent prognostic variables in patients with osteosarcoma. Besides, based on the risk score, age, gender, race, tumor site, and metastasis, we established a nomogram for forecasting the prognosis of the osteosarcoma patients. From this nomogram, we were able to predict the 1-, $3-$, and 5-year survival of osteosarcoma cases. The calibration plots verified that the signature could exactly appraise the survival of the osteosarcoma patients. Finally, in the past years, the underlying mechanisms of tumor susceptibility to stem cells have become a hot topic for research. On the foundation of the DEGs between two risk groups (low and high), we performed GO and KEGG analysis, which demonstrated that several tumorrelated biological functions were very affluent, such as the MAPK signaling pathway, Ras signaling pathway, chemokine signaling pathway, and apoptosis.

Despite the many advantages outlined above, our study has some drawbacks. First, there was lack of adequate cases and other clinicopathological information. Second, the performance of our prognostic signature lacked validation in more independent databases. Last, all of our results were on the foundation of

\section{REFERENCES}

Aydemir Coban, E., and Sahin, F. (2018). Cancer stem cells in metastasis therapy. Adv. Exp. Med. Biol. 1089, 97-113. doi: 10.1007/5584_2018_279

Bai, X. L., Zhang, Q., Ye, L. Y., Liang, F., Sun, X., Chen, Y., et al. (2015). Myocyte enhancer factor $2 \mathrm{C}$ regulation of hepatocellular carcinoma via vascular endothelial growth factor and Wnt/beta-catenin signaling. Oncogene 34, 40894097. doi: 10.1038/onc.2014.337

Bielack, S. S., Kempf-Bielack, B., Delling, G., Exner, G. U., Flege, S., Helmke, K., et al. (2002). Prognostic factors in high-grade osteosarcoma of the extremities or trunk: an analysis of 1,702 patients treated on neoadjuvant cooperative osteosarcoma study group protocols. J. Clin. Oncol. 20, 776-790. doi: 10.1200/ JCO.2002.20.3.776 the existing opening databases and lacked basic experimental verification as well as clinical information support.

\section{CONCLUSION}

In summary, we established a new prognostic signature based on eight stem cell-related genes. It proves that it is an independent factor that correlates with overall survival, hence, offering a novel orientation to forecast the prognosis of the osteosarcoma patients.

\section{DATA AVAILABILITY STATEMENT}

The datasets presented in this study can be found in online repositories. The names of the repository/repositories and accession number(s) can be found in the article/supplementary material.

\section{AUTHOR CONTRIBUTIONS}

BX designed the research study. BX, LL, ZC, YX, and YZ performed the literature search and statistical analysis. BX, AL, YX, PW, and CX interpreted the data and drafted the manuscript. $\mathrm{BX}$ and HL critically revised the manuscript. All authors read and approved the final manuscript.

\section{FUNDING}

This study was funded by including the National Natural Science Foundation of China (grant numbers 81502332 and 82072977), Research Project of Human Health Commission (grant number B2019162), Nature Science Foundation of Hunan (grant number 2019JJ50861), and Fundamental Research Funds for the Central Universities of Central South University (grant number 2021zzts0380).

\section{ACKNOWLEDGMENTS}

We appreciate The Cancer Genome Atlas (TCGA) database, GEO database, and MSigDB database.

Bishop, M. W., Janeway, K. A., and Gorlick, R. (2016). Future directions in the treatment of osteosarcoma. Curr. Opin. Pediatr. 28, 26-33. doi: 10.1097/MOP. 0000000000000298

Cao, Z. H., Yin, H. P., Jiang, N., and Yu, B. (2015). Association between ERCC1 and ERCC2 gene polymorphisms and chemotherapy response and overall survival in osteosarcoma. Genet. Mol. Res. 14, 10145-10151. doi: 10.4238/2015.August. 21.21

Chen, C., Xie, L., Ren, T., Huang, Y., Xu, J., and Guo, W. (2021). Immunotherapy for osteosarcoma: fundamental mechanism, rationale, and recent breakthroughs. Cancer Lett. 500, 1-10. doi: 10.1016/j.canlet.2020. 12.024

Chen, J., Liu, G., Wu, Y., Ma, J., Wu, H., Xie, Z., et al. (2019). CircMYO10 promotes osteosarcoma progression by regulating miR-370-3p/RUVBL1 axis to 
enhance the transcriptional activity of beta-catenin/LEF1 complex via effects on chromatin remodeling. Mol. Cancer 18:150. doi: 10.1186/s12943-019-1076-1

Cui, Y., Yu, S., Zhu, M., Cheng, X., Yu, Y., Tang, Z., et al. (2020). Identifying predictive factors of recurrence after radical resection in gastric cancer by RNA immune-oncology panel. J. Cancer 11, 638-647. doi: 10.7150/jca.38536

Dawood, S., Austin, L., and Cristofanilli, M. (2014). Cancer stem cells: implications for cancer therapy. Oncology 28, 1101-1107.

$\mathrm{Fu}, \mathrm{Z}$., Lu, C., Zhang, C., and Qiao, B. (2019). PSMA5 promotes the tumorigenic process of prostate cancer and is related to bortezomib resistance. Anticancer Drugs 30:e0773. doi: 10.1097/CAD.0000000000000773

Ginter, P. S., McIntire, P. J., Cui, X., Irshaid, L., Liu, Y., Chen, Z., et al. (2017). Folate receptor alpha expression is associated with increased risk of recurrence in triple-negative breast cancer. Clin. Breast Cancer 17, 544-549. doi: 10.1016/j. clbc.2017.03.007

He, H., Yi, Y., Cai, X., Wang, J., Ni, X., Fu, Y., et al. (2021). Down-regulation of EOMES drives T-cell exhaustion via abolishing EOMES-mediated repression of inhibitory receptors of T cells in liver cancer. J. Cell. Mol. Med. 25, 161-169. doi: $10.1111 /$ jcmm. 15898

He, M., Shen, P., Qiu, C., and Wang, J. (2019). miR-627-3p inhibits osteosarcoma cell proliferation and metastasis by targeting PTN. Aging 11, 5744-5756. doi: 10.18632/aging. 102157

Ho, X. D., Phung, P., Reimann, E., Prans, E., Koks, G., Maasalu, K., et al. (2017). Whole transcriptome analysis identifies differentially regulated networks between osteosarcoma and normal bone samples. Exp. Biol. Med. 242, 18021811. doi: $10.1177 / 1535370217736512$

Huang, M. J., Zhang, W., Wang, Q., Yang, Z. J., Liao, S. B., and Li, L. (2018). FOLR1 increases sensitivity to cisplatin treatment in ovarian cancer cells. J. Ovarian Res. 11:15. doi: 10.1186/s13048-018-0387-y

Hughes, D. P. (2009). How the NOTCH pathway contributes to the ability of osteosarcoma cells to metastasize. Cancer Treat. Res. 152, 479-496. doi: 10.1007/ 978-1-4419-0284-9_28

Iwakiri, S., Sonobe, M., Nagai, S., Hirata, T., Wada, H., and Miyahara, R. (2008). Expression status of folate receptor alpha is significantly correlated with prognosis in non-small-cell lung cancers. Ann. Surg. Oncol. 15, 889-899. doi: 10.1245/s10434-007-9755-3

Kim, M., Pyo, S., Kang, C. H., Lee, C. O., Lee, H. K., Choi, S. U., et al. (2018). Folate receptor 1 (FOLR1) targeted chimeric antigen receptor (CAR) T cells for the treatment of gastric cancer. PLoS One 13:e0198347. doi: 10.1371/journal.pone. 0198347

Kobel, M., Madore, J., Ramus, S. J., Clarke, B. A., Pharoah, P. D., Deen, S., et al. (2014). Evidence for a time-dependent association between FOLR1 expression and survival from ovarian carcinoma: implications for clinical testing. An ovarian tumour tissue analysis consortium study. Br. J. Cancer 111, 2297-2307. doi: $10.1038 / b j c .2014 .567$

Lytle, N. K., Barber, A. G., and Reya, T. (2018). Stem cell fate in cancer growth, progression and therapy resistance. Nat. Rev. Cancer 18, 669-680. doi: 10.1038/ s41568-018-0056-x

Nandy, S. B., and Lakshmanaswamy, R. (2017). Cancer stem cells and metastasis. Prog. Mol. Biol. Transl. Sci. 151, 137-176. doi: 10.1016/bs.pmbts.2017.07.007

Nassar, D., and Blanpain, C. (2016). Cancer stem cells: basic concepts and therapeutic implications. Annu. Rev. Pathol. 11, 47-76. doi: 10.1146/annurevpathol-012615-044438

Necela, B. M., Crozier, J. A., Andorfer, C. A., Lewis-Tuffin, L., Kachergus, J. M., Geiger, X. J., et al. (2015). Folate receptor-alpha (FOLR1) expression and function in triple negative tumors. PLoS One 10:e122209. doi: 10.1371/journal. pone. 0122209

Niu, J., Yan, T., Guo, W., Wang, W., and Zhao, Z. (2019). Insight into the role of autophagy in osteosarcoma and its therapeutic implication. Front. Oncol. 9:1232. doi: $10.3389 /$ fonc.2019.01232
Pu, Y., Zhao, F., Wang, H., and Cai, S. (2017). MiR-34a-5p promotes multichemoresistance of osteosarcoma through down-regulation of the DLL1 gene. Sci. Rep. 7:44218. doi: 10.1038/srep44218

Reimann, E., Koks, S., Ho, X. D., Maasalu, K., and Martson, A. (2014). Whole exome sequencing of a single osteosarcoma case-integrative analysis with whole transcriptome RNA-seq data. Hum. Genomics 8:20. doi: 10.1186/s40246-0140020-0

Reya, T., Morrison, S. J., Clarke, M. F., and Weissman, I. L. (2001). Stem cells, cancer, and cancer stem cells. Nature 414, 105-111. doi: 10.1038/351 02167

Rothzerg, E., Ho, X. D., Xu, J., Wood, D., Martson, A., Maasalu, K., et al. (2020). Alternative splicing of leptin receptor overlapping transcript in osteosarcoma. Exp. Biol. Med. 245, 1437-1443. doi: 10.1177/1535370220949139

Rothzerg, E., Xu, J., Wood, D., and Koks, S. (2021). 12 Survival-related differentially expressed genes based on the TARGET-osteosarcoma database. Exp. Biol. Med. [Epub ahead of print]. doi: 10.1177/15353702211007410

Sun, X., Tian, C., Zhang, H., Han, K., Zhou, M., Gan, Z., et al. (2020). Long noncoding RNA OIP5-AS1 mediates resistance to doxorubicin by regulating miR-137-3p/PTN axis in osteosarcoma. Biomed. Pharmacother. 128:110201. doi: 10.1016/j.biopha.2020.110201

van der Heijden, M., and Vermeulen, L. (2019). Stem cells in homeostasis and cancer of the gut. Mol. Cancer 18:66. doi: 10.1186/s12943-019-0962-x

Wakamatsu, T., Kakunaga, S., Takenaka, S., Outani, H., Hamada, K., Imura, Y., et al. (2019). Prognostic implication of adjuvant/neoadjuvant chemotherapy consisting of doxorubicin and ifosfamide in patients with extraskeletal osteosarcoma. Int. J. Clin. Oncol. 24, 1311-1319. doi: 10.1007/s10147-01901475- 1

Wang, S., Beeghly-Fadiel, A., Cai, Q., Cai, H., Guo, X., Shi, L., et al. (2018). Gene expression in triple-negative breast cancer in relation to survival. Breast Cancer Res. Treat. 171, 199-207. doi: 10.1007/s10549-018-4816-9

Yan, G. N., Lv, Y. F., and Guo, Q. N. (2016). Advances in osteosarcoma stem cell research and opportunities for novel therapeutic targets. Cancer Lett. 370, 268-274. doi: 10.1016/j.canlet.2015.11.003

Zhang, D., Qian, C., Wei, H., and Qian, X. (2020). Identification of the prognostic value of tumor microenvironment-related genes in esophageal squamous cell carcinoma. Front. Mol. Biosci. 7:599475. doi: 10.3389/fmolb.2020. 599475

Zhang, H., Liu, W., Wang, Z., Meng, L., Wang, Y., Yan, H., et al. (2018). MEF2C promotes gefitinib resistance in hepatic cancer cells through regulating MIG6 transcription. Tumori 104, 221-231. doi: 10.1177/03008916187 65555

Conflict of Interest: The authors declare that the research was conducted in the absence of any commercial or financial relationships that could be construed as a potential conflict of interest.

Publisher's Note: All claims expressed in this article are solely those of the authors and do not necessarily represent those of their affiliated organizations, or those of the publisher, the editors and the reviewers. Any product that may be evaluated in this article, or claim that may be made by its manufacturer, is not guaranteed or endorsed by the publisher.

Copyright (๑) 2021 Xiao, Liu, Chen, Li, Xia, Wang, Xiang, Zeng and Li. This is an open-access article distributed under the terms of the Creative Commons Attribution License (CC BY). The use, distribution or reproduction in other forums is permitted, provided the original author(s) and the copyright owner(s) are credited and that the original publication in this journal is cited, in accordance with accepted academic practice. No use, distribution or reproduction is permitted which does not comply with these terms. 